Pulsed operation of high-power light emitting diodes for imaging flow velocimetry

This article has been downloaded from IOPscience. Please scroll down to see the full text article.

2010 Meas. Sci. Technol. 21075402

(http://iopscience.iop.org/0957-0233/21/7/075402)

View the table of contents for this issue, or go to the journal homepage for more

Download details:

IP Address: 129.247.247.239

The article was downloaded on 08/06/2010 at $15: 23$

Please note that terms and conditions apply. 


\title{
Pulsed operation of high-power light emitting diodes for imaging flow velocimetry
}

\author{
C Willert ${ }^{1}$, B Stasicki ${ }^{2}$, Klinner $^{1}$ and S Moessner $^{1}$ \\ ${ }^{1}$ Engine Measurement Techniques, Institute of Propulsion Technology, German Aerospace Center \\ (DLR), 51170 Köln, Germany \\ ${ }^{2}$ Experimental Techniques, Institute of Aerodynamics and Flow Technology, German Aerospace Center \\ (DLR), 37073 Göttingen, Germany \\ E-mail: chris.willert@dlr.de
}

Received 8 January 2010, in final form 16 April 2010

Published 8 June 2010

Online at stacks.iop.org/MST/21/075402

\begin{abstract}
High-powered light emitting diodes (LED) are investigated for possible uses as light sources in flow diagnostics, in particular, as an alternative to laser-based illumination in particle imaging flow velocimetry in side-scatter imaging arrangements. Recent developments in solid state illumination resulted in mass-produced LEDs that provide average radiant power in excess of $10 \mathrm{~W}$. By operating these LEDs with short duration, pulsed currents that are considerably beyond their continuous current damage threshold, light pulses can be generated that are sufficient to illuminate and image micron-sized particles in flow velocimetry. Time-resolved PIV measurements in water at a framing rate of $2 \mathrm{kHz}$ are presented. The feasibility of LED-based PIV measurements in air is also demonstrated.
\end{abstract}

Keywords: particle image velocimetry, PIV, light emitting diode, LED, pulsed light source, time-resolved PIV, flow diagnostics, flow visualization

(Some figures in this article are in colour only in the electronic version)

\section{List of symbols}

$A_{\text {lum }}$ emitter luminescent area,

$f \quad$ focal length,

$f_{\#} \quad$ lens aperture $(f$-number $)$,

$I_{\mathrm{f}, \mathrm{cw}}$ continuous forward current,

$I_{\mathrm{f}, \max }$ maximum continuous forward current,

N.A. numerical aperture,

$P_{\text {diss }}$ dissipated power,

$\lambda_{\mathrm{o}}$ dominant emission wavelength,

$\Phi_{v} \quad$ luminous flux in lumen $(\mathrm{lm})$,

$\Phi_{r} \quad$ radiometric flux in watt $(\mathrm{W})$,

$\Delta t \quad$ delay between two illumination pulses (for PIV),

$\tau_{\mathrm{p}} \quad$ pulse duration,

$\tau_{\text {rise }} \quad$ rise time.

\section{Introduction}

While earlier generations of LEDs were primarily used as indicators in everyday electronics, recent developments have resulted in mass-produced devices whose light output rivals that of conventional light sources such as incandescent light bulbs and now find an increased range of applications ranging from architectural lighting to projection systems and automotive headlights. In the framework of flow diagnostics, LEDs promise a number of attractive advantages in comparison to lasers and therefore deserve closer investigation. Aside from the dramatically reduced cost of procurement and considerably longer lifetime, an LED provides incoherent light over a rather wide wavelength range (typ. $\pm 10-30 \mathrm{~nm}$ ) which alleviates many issues related to speckle artifacts found in laser-based illumination. If operated in pulsed mode, the luminosity signal of an LED is extremely stable, both in terms of intensity and spatial intensity distribution. On the other hand the uncollimated nature of the emitted light presents several challenges if the LED is to be used as alternative light source to lasers based in flow diagnostics. 
Another interesting characteristic and a main point of emphasis of the present work is that LEDs can be operated significantly beyond their damage threshold using high-current, short duration pulses. In this case, the temperature within the substrate (junction temperature) stays below the damage threshold while the photon generation per time unit is approximately increased in proportion to the current increase [1,2]. This makes the LED particularly interesting for utilization as a pulsed light source in imagebased diagnostics. The literature reports the use of LEDs in schlieren imaging [3], bubble shadowgraphy [4-6], shadow velocimetry [7], PIV [8] as well as micro-PIV [9]. If operated in a forward scatter shadowgraphy arrangement short duration pulses of $\tau_{p}<250 \mathrm{~ns}$ enable high-speed shadowgraphy in the $100 \mathrm{kHz}$ range of kerosene sprays at high magnification [10]. Short laser as well as LED light pulses can be used as a back illumination for the visualization and recording of the liquid micro-jet disintegration $[11,12]$. The present work focuses on side-scatter illumination geometries commonly used in PIV and PTV which require significantly higher illumination intensities.

The following article is arranged in two main parts. The first part deals with the characteristics of high-power LEDs when subjected to pulsed drive currents, including measurements of the increased light flux as a function of drive current, spectral emission characteristics and damage thresholds. The second part investigates the possible use of LEDs in side-scatter imaging configurations both in air and in water flows.

\section{Overview of high-current LEDs}

Table 1 lists the characteristics of several representative LEDs tested in the course of the feasibility study. Mainly green LEDs were investigated, in part because present day imaging sensors exhibit peak quantum efficiencies in the yellow/green range $\left(530<\lambda_{\mathrm{o}}<550 \mathrm{~nm}\right)$, similar to the response of the human eye. The listed devices represent two different types of LEDs: the first three, available from Philips Lumileds, are designed for general illumination purposes and contain rather small luminous emission chips which are encapsulated in a lensshaped clear dome ${ }^{3}$. The other two devices, available from Luminus Devices Inc., are specifically designed for projection systems. Here the luminescent substrate is covered by a thin glass window similar to an imaging sensor. Contrary to most commonly available LEDs these devices are essentially surface emitters with a nearly constant intensity distribution per unit area. This is achieved by a photonic lattice attached to the surface of the emitter which channels the light through micronsized, surface-normal holes. Because of this, the radiation pattern is more collimated than for conventional high-current LEDs.

\section{LED characteristics in pulsed operation}

A central aspect of the current investigation was the pulsed operation of LEDs with a high current over a short duration.

3 At the end of year 2009 Philips Lumileds discontinued the manufacture of the tested LED devices, replacing them with devices of similar performance but different packaging.
Table 1. Specifications of selected high-current LEDs.

\begin{tabular}{lcclclc}
\hline Emitter & $\begin{array}{l}I_{\mathrm{f}, \mathrm{cw}} \\
(\mathrm{A})\end{array}$ & $\begin{array}{l}\Phi_{V} \\
(\mathrm{~lm})\end{array}$ & $\begin{array}{l}\lambda_{\mathrm{o}} \\
(\mathrm{nm})\end{array}$ & $\begin{array}{l}I_{\mathrm{f}, \max }(\mathrm{A}) \\
A_{\text {lum }} \\
\left(\mathrm{mm}^{2}\right)\end{array}$ & $\begin{array}{l}P_{\text {diss }} \\
(\mathrm{W})\end{array}$ \\
\hline LXHL-PD09 & 1.4 & 140 & 627 & 1.54 & $\approx 1.5 \times 1.5$ & 3 \\
LXHL-PL09 & 1.4 & 110 & 590 & 1.54 & $\approx 1.5 \times 1.5$ & 5 \\
LXHL-PM02 & 0.7 & 160 & 530 & 1.00 & $\approx 2 \times 2$ & 5 \\
CBT-40 (green) & 5.9 & 625 & 528 & 12 & $2.09 \times 1.87$ & 25 \\
CBT-120 (green) & 18 & 2100 & 528 & 36 & $4.6 \times 2.6$ & 77 \\
\hline
\end{tabular}

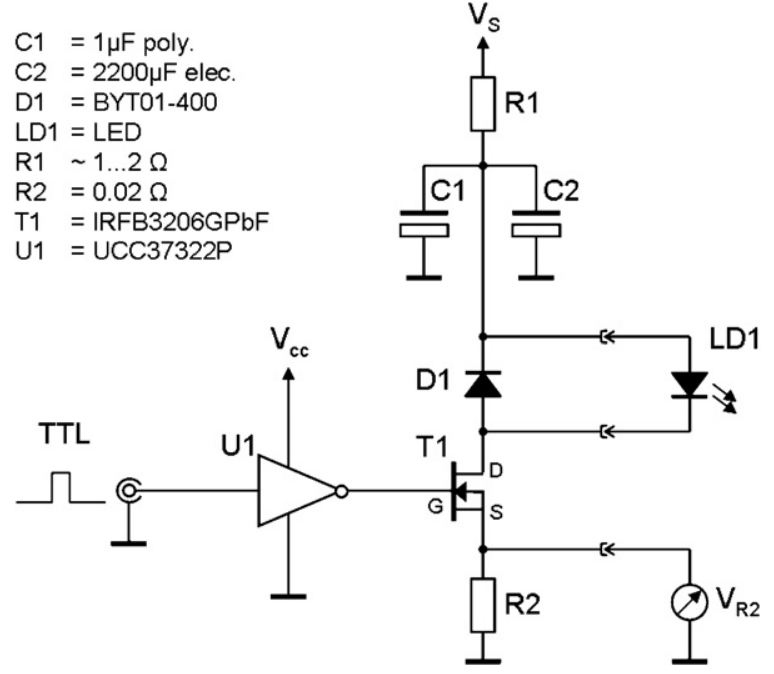

Figure 1. Example of driver circuit used by the authors for pulsed operation of LEDs.

This was achieved using electronic circuitry which is schematically outlined in figure 1 . The operation of the current pulser is very straightforward: a bank of capacitors $(\mathrm{C} 1, \mathrm{C} 2)$ with low internal resistance (ESR) is first charged through the power supply $V_{\mathrm{S}}$ (typ. 20-50 V). The MOSFET power transistor (T1) is triggered via a MOSFET driver circuit (U1) which has a TTL compatible input. The LED (LD1) cathode is connected to the drain of the power transistor whose source is connected to ground via a low resistance power resistor (R2, typ. $0.01 \Omega-0.1 \Omega$ ). In this configuration the circuit acts as a switched constant-current source for the load, that is, the LED. The voltage drop across this resistor $V_{\mathrm{R} 2}$ is used to estimate the current flowing through the LED. Diode D1 protects the LED from potentially damaging reverse currents that arise during the rapid switching transients of the circuit. The purpose of resistor R1 is to limit the charging current of the capacitor bank in between the current pulses and it acts as a rudimentary method of overcurrent protection in case the LED is accidentally operated in continuous mode. Short lead lengths with high cross sections between capacitors $\mathrm{C} 1, \mathrm{C} 2$, transistor $\mathrm{T} 1$, diode $\mathrm{D} 1, \mathrm{LED} \mathrm{LD} 1$ and ground are essential to obtain good performance. Due to the high pulsed currents and rise times in the microsecond range even short connecting wires result both in significant voltage drops between the electronic components as well as unnecessary inductive loading of the circuit.

The measurements of pulsed LED operation presented in the following sections were performed using both selfdesigned and commercially available current pulsers. The 

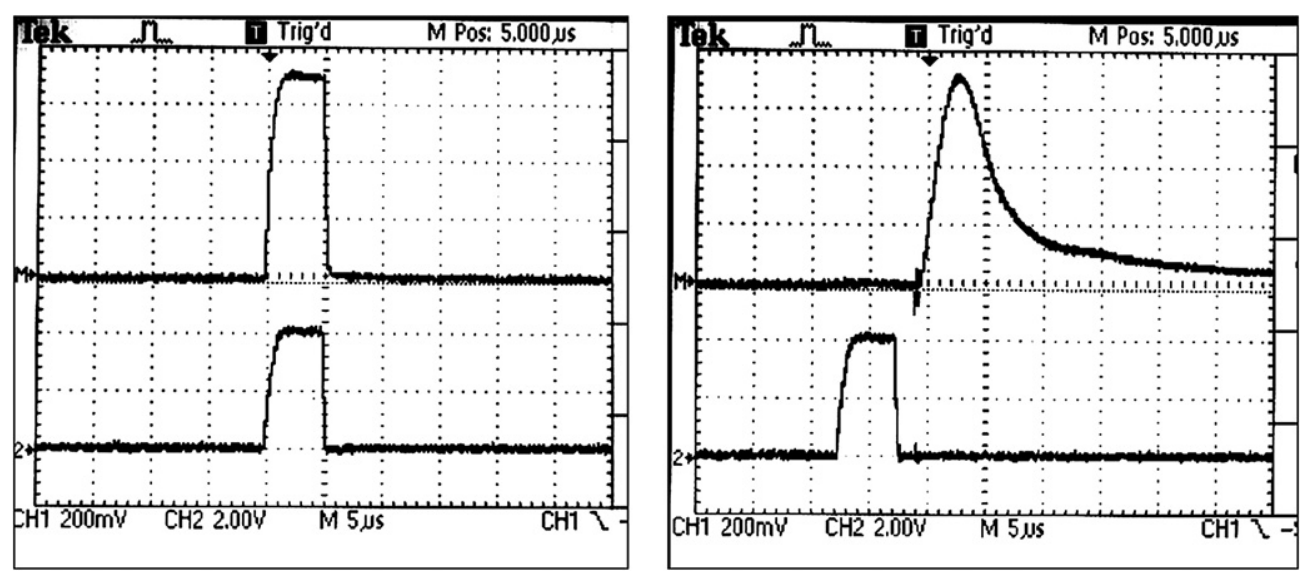

Figure 2. Time trace of light emission as detected by a photodiode (upper trace) for a high-power LED (left) and a conventional xenon flash lamp (right) in response to a trigger pulse (lower trace).

commercial device (LDP-V 50-100 V3, PicoLAS GmbH) was specifically designed for the pulsed operation of laser diodes and is capable of providing current pulses of up to $I_{\mathrm{f}}=50 \mathrm{~A}$ at durations of up to $\tau_{\mathrm{p}}=10 \mu \mathrm{s}$ with corresponding rise times of $\tau_{\text {rise }} \leqslant 4$ ns. Due to a limited capacitance available on the supply side of the unit, longer pulses $\tau_{\mathrm{p}}>1 \mu$ s exhibit a noticeable exponential decay of the LED drive current and resulted in a corresponding exponential decrease in LED light output during the pulse. This issue was solved with a selfdesigned driver whose slower rise time (typ. $\tau_{\text {rise }} \approx 100 \mathrm{ns)}$ ) is acceptable given that the studied range of applications makes use of pulses in the $1 \mu \mathrm{s}<\tau_{\mathrm{p}}<20 \mu \mathrm{s}$ range. This circuit is capable of delivering pulsed currents in excess of $200 \mathrm{~A}$ using the parts specified in figure 1 .

The response of the LED to a current pulse is provided in figure 2(left), where the lower time trace corresponds to the input TTL pulse while the upper is a measurement of the emitted light as detected by a photodiode. Two features are worth remarking in comparison to the response of a conventional xenon flash lamp (EG\&G) shown in figure 2(right). First, the duration of the LED light emission can be precisely controlled by the width of the input drive pulse. In contrast, the flash lamp intensity is not correlated with the input pulse modulation and cannot be shortened or lengthened in a simple manner. Second, the rise time of the $\operatorname{LED}\left(\tau_{\text {rise,LED }}<1 \mu \mathrm{s}\right)$ is significantly shorter than for the examined flash lamp ( $\left.\tau_{\text {rise,FL }} \approx 4 \mu \mathrm{s}\right)$. In a similar manner, the decay time of the LED emission strictly follows the drive pulse while the flash lamp's intensity decays in an exponential fashion over several tens of microseconds. Finally there is no significant lag between the rising edge of input drive pulse and the light flash emitted by the LED while the emission of the flash lamp is delayed by about $8 \mu \mathrm{s}$. The reason for this delay is not exactly known and varies from model to model.

Figure 3 illustrates the increased luminosity of two highpower LEDs that are driven with short-duration current pulses (here $\tau_{p}=1 \mu \mathrm{s}$ at $1 \mathrm{kHz}$ ). In both cases the LEDs are driven up to maximum currents in the $30 \mathrm{~A}$ range, which is more than a factor 20 higher than the rated forward current $I_{f}$ for the red LED. For the green LED the rated current is exceeded

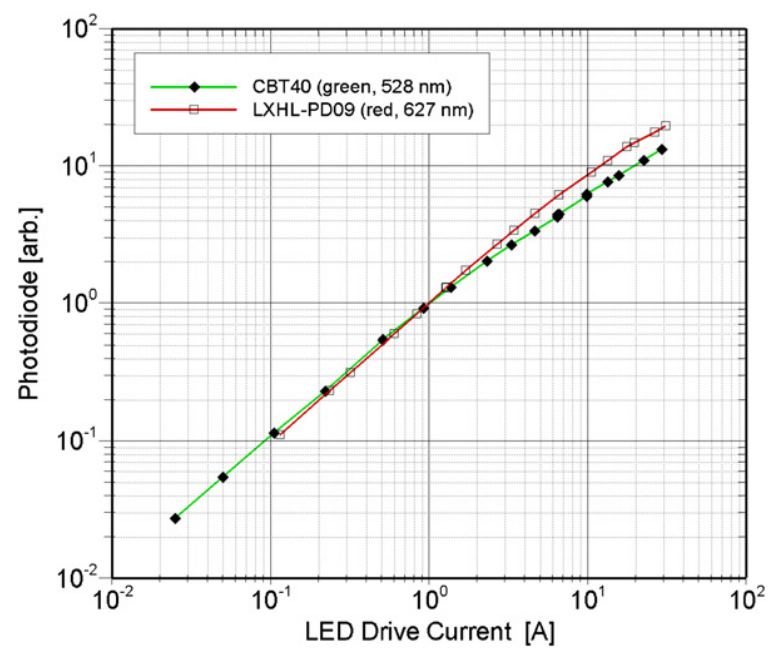

Figure 3. Luminosity of high-power LEDs (LXHL-PD09, Philips Lumileds; CBT-40, Luminus Devices) driven with $1 \mathrm{kHz}$ current pulses of $\tau_{p}=1 \mu$ s duration.

by a factor of 5 , suggesting further increase without damage to the device. In fact none of the tested devices suffered any noticeable damage when operated in pulsed mode at these current levels and low duty cycle (less than 1:100).

The data shown in figure 3 confirm the known increase of luminous flux $\Phi_{v}$ with an increasing drive current $I_{f}$. For the red LED the increase of forward current leads to a proportional increase for low drive currents. At higher currents the efficiency factor or proportionality reduces to about 0.4 . The green LED (CBT-40) exhibits a similar behavior for low currents but decreases to about 0.35 at currents beyond $I_{f}=10 \mathrm{~A}$. This indicates a decreasing proportionality with increasing drive currents due to saturation effects. Not shown are data for the $5 \mathrm{~W}$ green LED (LXHL-PM02) for which the proportionality decreases to 0.2 at currents beyond $I_{f}=30 \mathrm{~A}$. In comparison the high-power device CBT-120, which has a three times larger luminescent area than the CBT-40, exhibits a proportionality of 0.47 at $I_{f}=40 \mathrm{~A}$, indicating considerable margins for operation at even higher currents. This was confirmed by additional measurements performed on the 


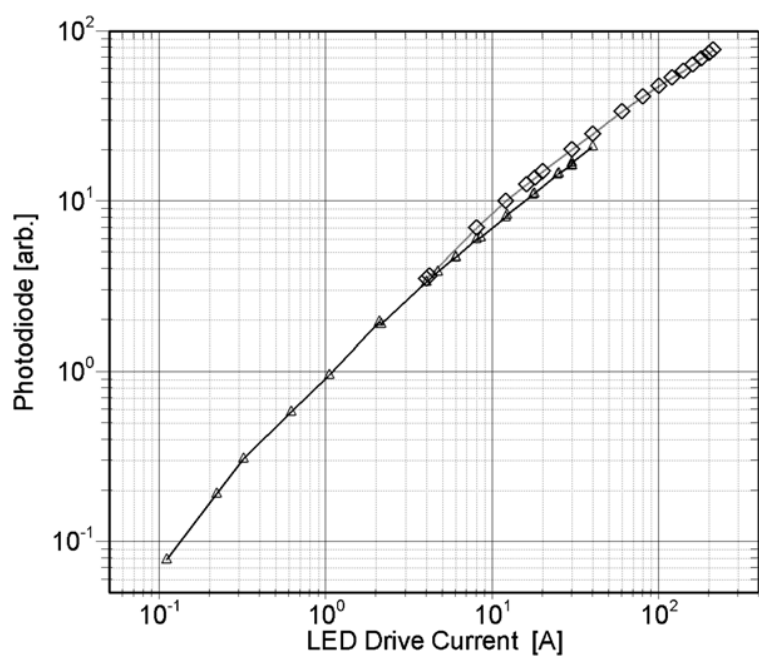

Figure 4. Luminosity of a high-power green LED (CBT-120, Luminus Devices) driven with current pulses of duration $1 \mu \mathrm{s}$ at $1 \mathrm{kHz}(\triangle)$ and $10 \mu \mathrm{s}$ at $25 \mathrm{~Hz}(\diamond)$.

CBT-120 LED and reproduced in figure 4. The data were obtained using a self-designed driver similar to the one shown in figure 1 capable of delivering $\tau_{p}=10 \mu$ s pulses at a current of up to $I_{f}=220$ A. Driving this LED at a current of $I_{f}=200 \mathrm{~A}$ instead of its rated current of $I_{f}=18 \mathrm{~A}$ increases its light emission by a factor of $\sim 5.5$.

It should be noted that the curves shown in figures 3 and 4 have not been corrected for the wavelength-dependent response of the detector photodiode (Thorlabs, PDA10A). As will be described in the following section the green LEDs have a tendency of emitting shorter wavelength light when subjected to increasing forward currents. Since the photodiode's responsivity decreases toward the blue-green spectral range the relative luminuous flux for the green LEDs may actually be higher than the measured values presented in figures 3 and 4. On the other hand, most CCD and CMOS camera sensors also have reduced responsivity in this spectral range, such that these measurements are representative of the relative signal gain at the image sensor level when overdriving LEDs.

Measurements of the type shown in figures 3 and 4 allow an approximate extrapolation of luminous flux (or effective pulse energy) at even higher currents. The smaller highcurrent devices LXHL-PD09 (red) and LXHL-PM02 (green) could be driven at pulsed currents of $50 \mathrm{~A}$ without immediate damage, exceeding the rated current by a factor of 30 and 70 , respectively. This suggests that the stronger devices CBT-40 and CBT-120 can be driven at significantly higher currents in the 100-200 A range. The data sheet for the CBT-120 quotes a radiometric flux $\Phi_{r}=6.3 \mathrm{~W}\left(\Phi_{v}=3100 \mathrm{~lm}\right)$ when driven at a pulsed current of $I_{f}=30 \mathrm{~A}$ at $240 \mathrm{~Hz}$ and a $50 \%$ duty cycle [13]. The effective pulse power then is $\Phi_{r}=12.6 \mathrm{~W}$ for the duration of the current pulse. Assuming a conservative proportionality of $35 \%$ light output increase with respect to increasing current - as indicated in figure 3-this would result in an effective pulse power of close $60 \mathrm{~W}$ at $I_{f}=200$ A drive current. This corresponds to a pulse energy of $60 \mu \mathrm{J}$ for a
$1 \mu$ s pulse. As will be demonstrated later this is sufficient to perform reliable high-speed PIV measurements in water.

\subsection{Spectral characteristics}

A side effect of the pulsed operation of LEDs is that the emission wavelength may shift considerably as the current is increased, generally toward shorter wavelengths. This effect is shown in figure 5 for two LED emitters. The drive pulses are approximately chosen to be of similar integral input power to maintain an average junction temperature. While the amber LED (LXHL-PL09) shows only a minor frequency shift of about $\Delta \lambda=2 \mathrm{~nm}$ toward longer wavelengths, the green device (CBT-40) shifts by more than $\Delta \lambda=30 \mathrm{~nm}$ toward a cyan color. The effect is characteristic for the respective junction materials that the devices are made of, typically GaAsP or AlInGaP for the red and orange luminescence and $\mathrm{InGaN}$ for the green and blue LEDs [14]. The spectral width increases slightly for both devices as the drive current increases. In general, the green device has a much wider emission spectrum which is also attributed to the junction material (so-called alloy broadening [14]). While the frequency shift may not be an issue for many velocimetry applications it nonetheless may be of interest when color-selective cameras or filters are used. Wavelength variations may also influence measurements of fluorescence.

\subsection{Damage thresholds}

After irreversibly damaging several devices in the early stages of this investigation a systematic study was performed to determine the safe operating envelope of the LED used most frequently by the authors (CBT-120, green). This was achieved by fixing the forward pulse current $I_{f}$ on the diode and gradually increasing the pulse width until the LED failed. In all cases the LEDs failed due to melting of the bond wires connecting the anode side of the die to the supply line. The measurement results are plotted in figure 6 and were obtained for a pulsing frequency of $25 \mathrm{~Hz}$. Data on previously observed failures have been included (e.g., $2 \mu$ s at $350 \mathrm{~A}$ ). The safe operating envelope-indicated as a dotted line-exhibits three main operation regimes. For pulse widths below $5 \mu \mathrm{s}$ the maximum pulse current is limited to $I_{f} \approx 250 \mathrm{~A}$. For pulse widths in the range $5 \mu \mathrm{s}<\tau_{\mathrm{p}}<200 \mu \mathrm{s}$ the damage threshold exhibits a log-linear trend. For pulses $\tau_{\mathrm{p}} \geqslant 200 \mu$ s the damage threshold is in agreement with the maximum current rating of $I_{\mathrm{f}, \max }=36$ A provided in the data sheet for the device [13] and shown as a dashed line in figure 6 .

The maximum pulsed current limit of 250 A suggests that the bonding wires fail immediately much like a conventional fuse. In the intermediate range for pulse widths of $5 \mu \mathrm{s}<$ $\tau_{\mathrm{p}}<200 \mu \mathrm{s}$ the heating of the die due to high current fluxes is believed to additionally heat the bonding wires, which raises their resistance and limits the maximum sustainable current. This seems to be supported by the fact that all bonding wires were melted on the die side of the connection, leaving the supply side unaffected. LEDs damaged due to short current pulses exhibit bonding wires that are nearly completely melted with significant deposits on the front cover 

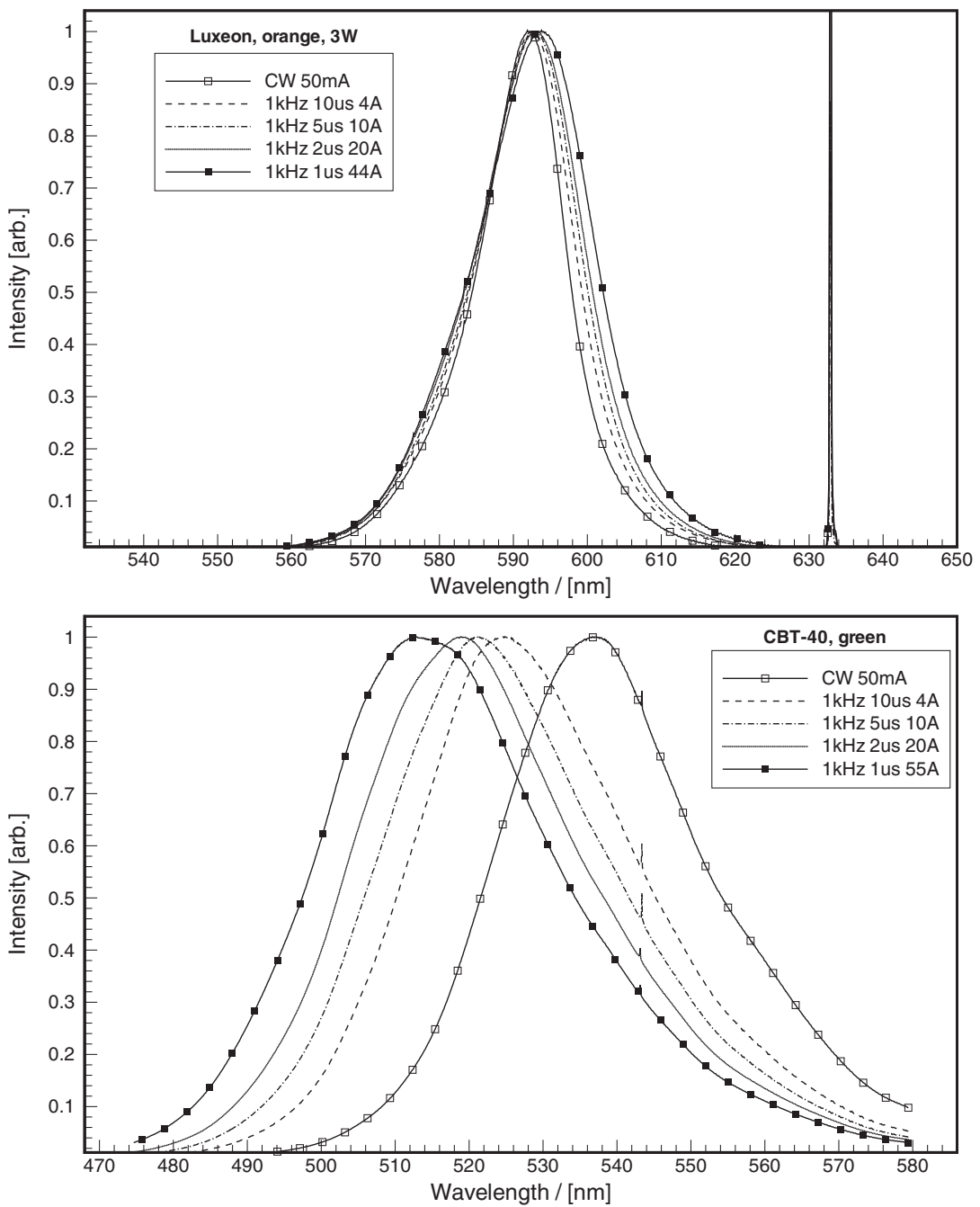

Figure 5. Spectral intensity distribution of LEDs operating in continuous and pulse modes. Top: LXHL-PL09 (amber); bottom: CBT-40 (green).

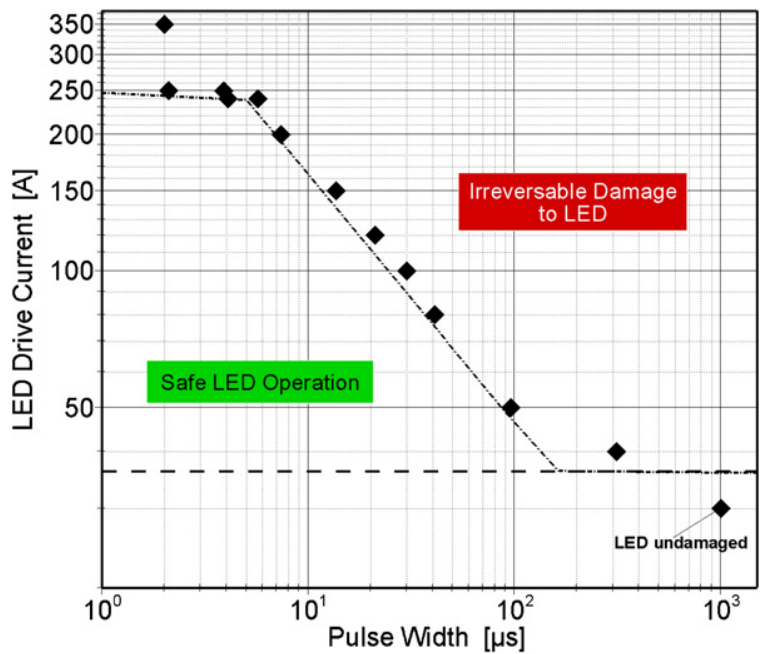

Figure 6. Experimentally obtained pulsed current damage threshold for a CBT-120 (green) LED. The dashed horizontal line indicates the maximum rated forward current (36 A) quoted in the device's data sheet. glass window.For pulse widths $\tau_{\mathrm{p}}>200 \mu$ s the LED will fail due to thermal damage in the die itself, typically at internal junction temperatures exceeding $150{ }^{\circ} \mathrm{C}$. These observations suggest that thicker or more bonding wires may actually allow the diode to withstand even higher, short-duration pulsed currents. This seems supported by the fact that the Luxeon devices (LXHL-PM02, LXHL-PL09) sustained continuous $1 \mathrm{kHz}$ pulses of $\tau_{\mathrm{p}}=1 \mu \mathrm{s}$ at forward currents $I_{f} \geqslant 40 \mathrm{~A}$ which is more than 50 times the rated current for the LXHLPM02 device. In comparison, the CBT-120 devices failed at about 14 times the rated continuous forward current.

Another possible cause for LED damage is too short a rise time of the pulse current. Even though the LED can withstand a continuous train of $200 \mathrm{~A}$ pulses of $\tau_{p}=7.5 \mu \mathrm{s}$ with rise times of about $\tau_{\text {rise }} \approx 1 \mu \mathrm{s}$, it will be immediately damaged when the rise time is shortened below $0.5 \mu \mathrm{s}$. The data sheet for the device also recommends rise times of greater than $0.5 \mu$ s from $10 \%$ to $90 \%$ of forward current [13].

While the devices can be damaged due to excessive current overdriving, increased ageing is likely to occur, resulting in 


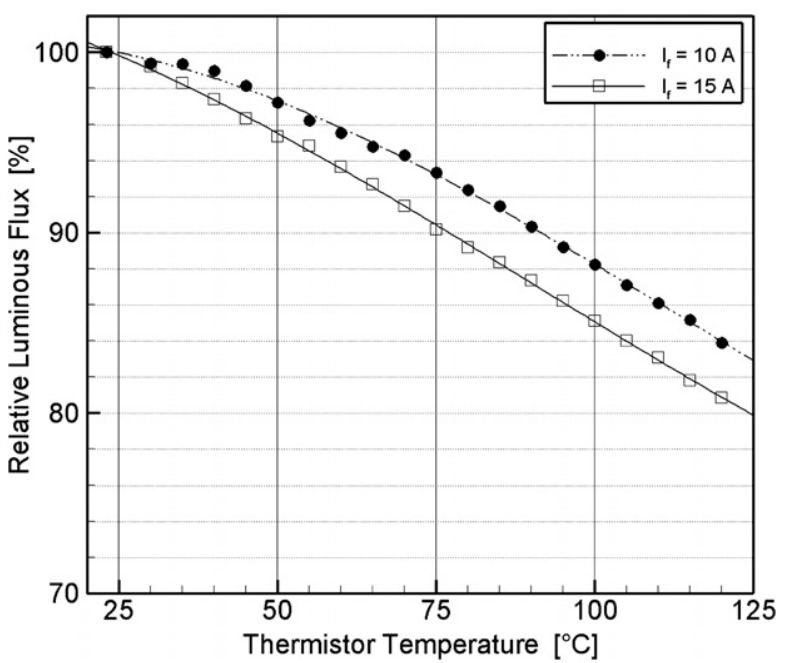

Figure 7. Luminous flux versus die temperature for a CBT-120 (green) LED running in $\mathrm{CW}$ mode $\left(I_{f}=15 \mathrm{~A}, P_{\text {diss }}=93 \mathrm{~W}\right)$.

a gradual reduction of light emission, but this has not been noticed or investigated by the authors. Driving the LEDs at high currents briefly increases the junction temperature which strains the crystal, causing defects in its lattice which then no longer contribute to light generation. With increasing temperatures the luminous flux from the LED also decreases such that active cooling can in fact both increase the light output (figure 7) as well as increase the lifetime of the LED itself. A convenient way of cooling the LED is offered through the use of commercially available cooling assemblies for personal computer CPUs along with copper plate to function as a heat spreader between LED and heat sink.

\section{PIV using LED}

As mentioned in section 1, the literature reports a number of earlier PIV applications involving LED clusters as light sources [4, 5, 7-9]. In these applications the LEDs were rarely overdriven to levels reported here and generally were operated in an in-line imaging arrangement or relied on large particles to achieve sufficient light scattering.

The present LED-PIV investigations focused on using single LEDs to illuminate particles in a side-scatter arrangement which is commonly used in planar PIV. Initially volume illumination was used in which the luminescent area of the LED is projected into the flow under investigation using condenser lenses. A combination of high magnification and large lens aperture limited the depth of field in the sub-millimeter regime and permitted two-component PIV measurements on an area of about $10 \times 7.5 \mathrm{~mm}^{2}$. A $5 \mathrm{~W}$ class green LED (LXHL-PM02) was pulsed at $I_{f}=2$ A for $\tau_{\mathrm{p}}=20 \mu \mathrm{s}$. PIV measurements were reliable using $10 \mu \mathrm{m}$ particles in water, imaged by a cooled, double-shutter CCD camera.

\subsection{LED-PIV measurements of an air flow}

Motivated by the initial PIV measurements in water flows, later PIV experiments made use of stronger, projection type LEDs (CBT-40, CBT-120) that could be driven at much higher currents and due to their larger luminescent areas provided considerably more light per unit time. These devices were used to test the feasibility of imaging micron-sized particles in air. Figure 8 shows a PIV recording imaging a $9.5 \times 7 \mathrm{~mm}^{2}$ portion of a free jet in air seeded with 1-3 $\mu \mathrm{m}$ glycerin droplets. Volume illumination was provided by the CBT-120 LED device using $30 \mathrm{~A}$ pulses of $\tau_{\mathrm{p}}=2 \mu$ s duration. The pulse delay was $\Delta t=15 \mu \mathrm{s}$. Arranged in a $45^{\circ}$ forward scattering with respect to the LED light a macro lens (Nikon Nikkor MF $55 \mathrm{~mm} / f_{\#} 2.8$ ) with aperture set at $f_{\#} 4$ imaged the flow onto the actively cooled sensor $(1376 \times 1040$ pixel $)$ of a double-shutter CCD camera (Sensicam QE, PCO AG) using a magnification of approximately $1: 1$. Assuming a circle of confusion of 5 pixel the depth of field is estimated to be $500 \mu \mathrm{m}$. The processed PIV data set shown in figure 9 was analyzed using sampling areas of $48 \times 48$ pixel $\left(320 \times 320 \mu \mathrm{m}^{2}\right)$.

While these PIV measurements demonstrate the feasibility of imaging micron-sized droplets in air at moderate
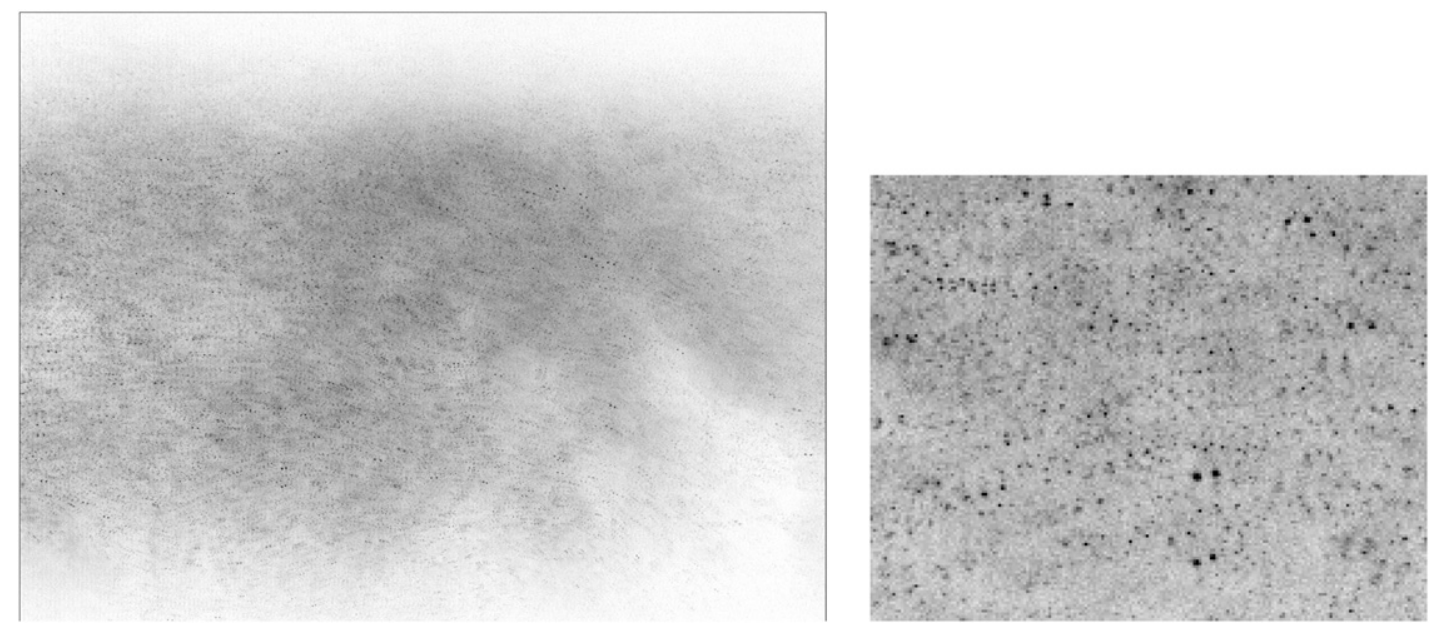

Figure 8. PIV recording of an air flow obtained with LED illumination in forward scatter shown with inverted intensity scale (black $=$ bright). Magnified sub-region of image to the left (area $\approx 1.5 \times 1.2 \mathrm{~mm}^{2}$ ) (right). 


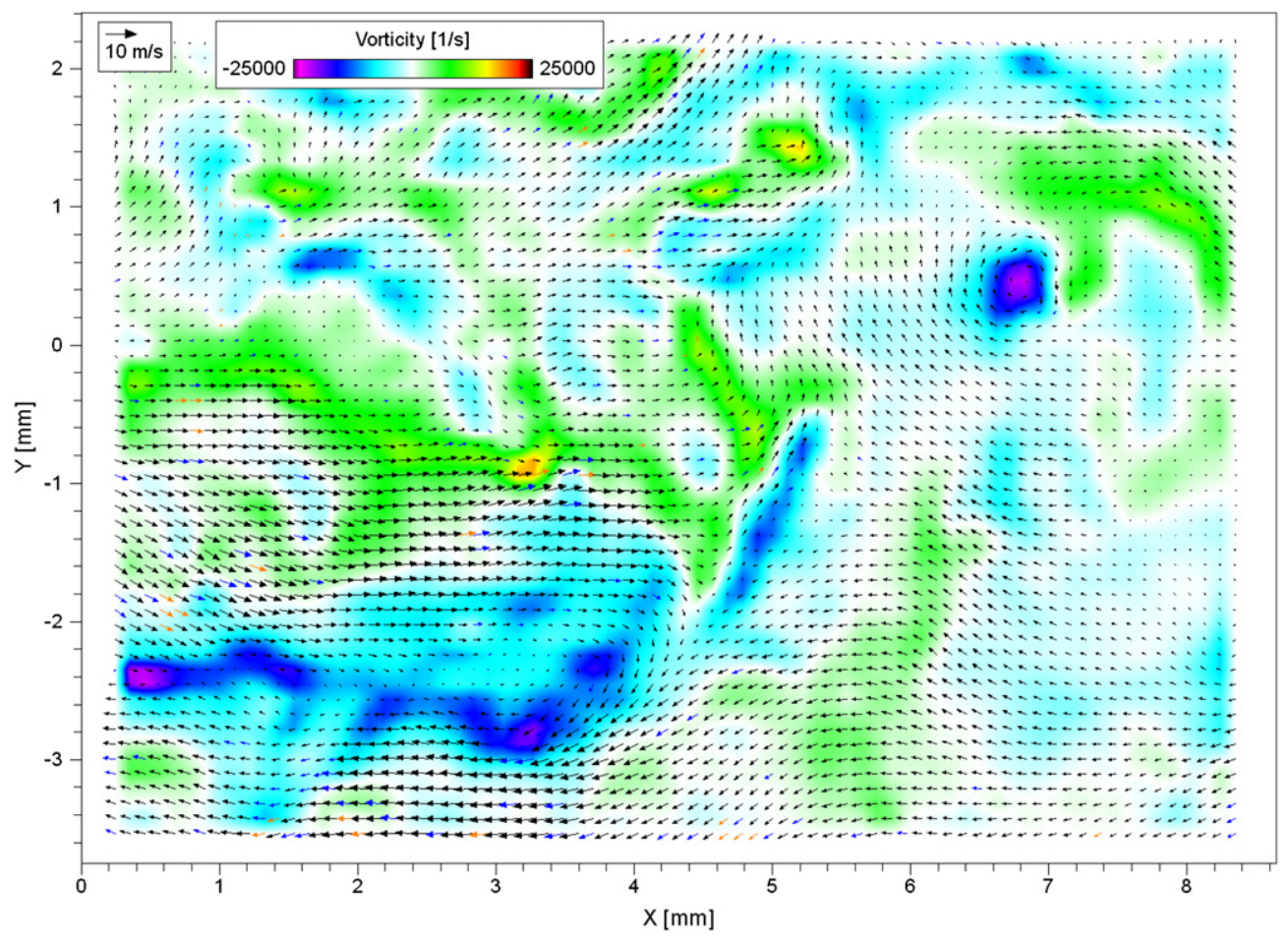

Figure 9. Processed PIV data set corresponding to figure 8 obtained with LED illumination. For clarity purposes a constant horizontal flow component of about $7 \mathrm{~m} \mathrm{~s}^{-1}$ has been subtracted. Orange vectors indicate interpolated data, and blue vectors are displacement data obtained from lower order correlation peaks.
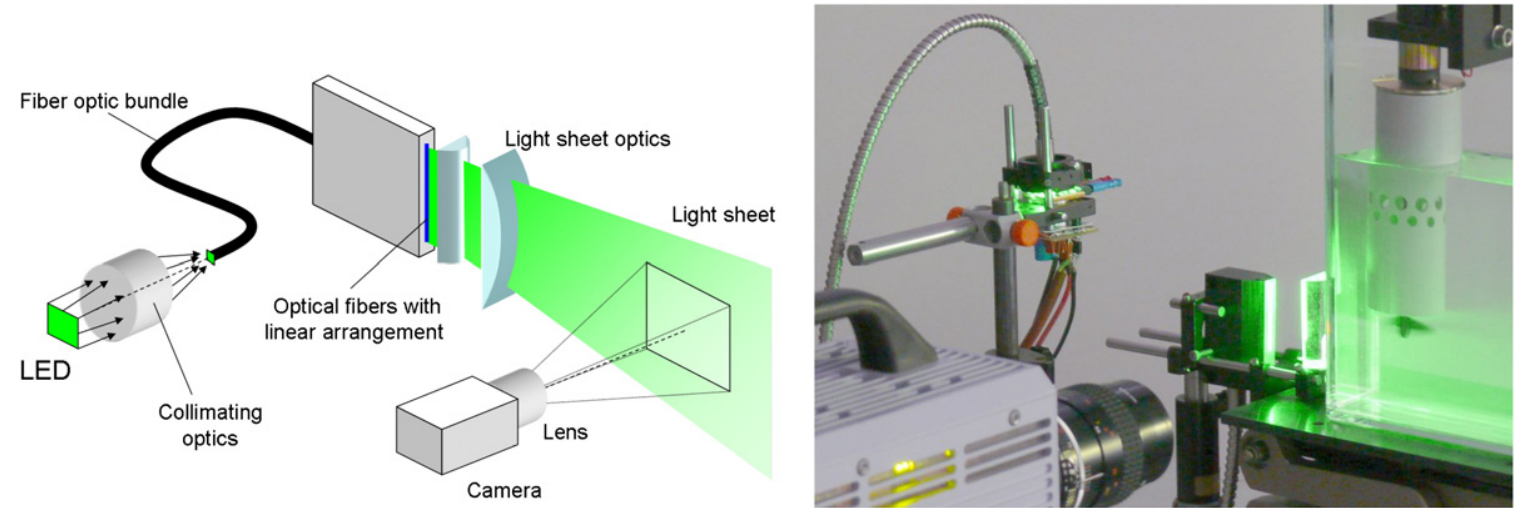

Figure 10. Schematic of LED-based light sheet illumination using a fiber optic line-light assembly (left). Photograph of a high frame rate LED-PIV setup on a small propeller in water (right).

velocities using rather short illumination pulses, the method of volume illumination has the drawback of introducing significant background signal due to the light scattered by out-of-focus particles. This reduces image contrast and poses a common problem for PIV applications in general. Therefore possibilities of converting the highly divergent light of the LEDs into a light sheet were investigated in a further iteration.

\subsection{LED-PIV measurements of a water flow}

The most promising experiments were performed when the LED light was bundled into a light sheet by means of a fiberoptic illumination system, also known as line light (e.g., DolanJenner Industries, Model QF2036). As illustrated in figure 10 the entry side of the fiber bundle is round ( $3 \mathrm{~mm}$ diameter) while the fibers at the distal end are arranged along a straight line of $38 \mathrm{~mm}$ height and $0.5 \mathrm{~mm}$ thickness. A light sheet can then be formed by projecting this line into the area under investigation using a short focal length cylindrical lens (here $f=25 \mathrm{~mm}$ ).

Figure 11 provides a sample PIV result from a time series of the turbulent water flow downstream of a small propeller acquired at a frame rate of $2 \mathrm{kHz}$ using pulsed LED illumination and a high-speed CMOS camera (PCO.DIMAX, PCO AG). Fitted with $f=55 \mathrm{~mm} f_{\# 2} 2.8$ lens (Nikon Nikkor MF) the camera's image magnification was about 40 pixel $\mathrm{mm}^{-1}$. The water was seeded with $10 \mu \mathrm{m}$ hollow glass spheres (Conduct-o-Fil, Potters Industries Inc.). The light 


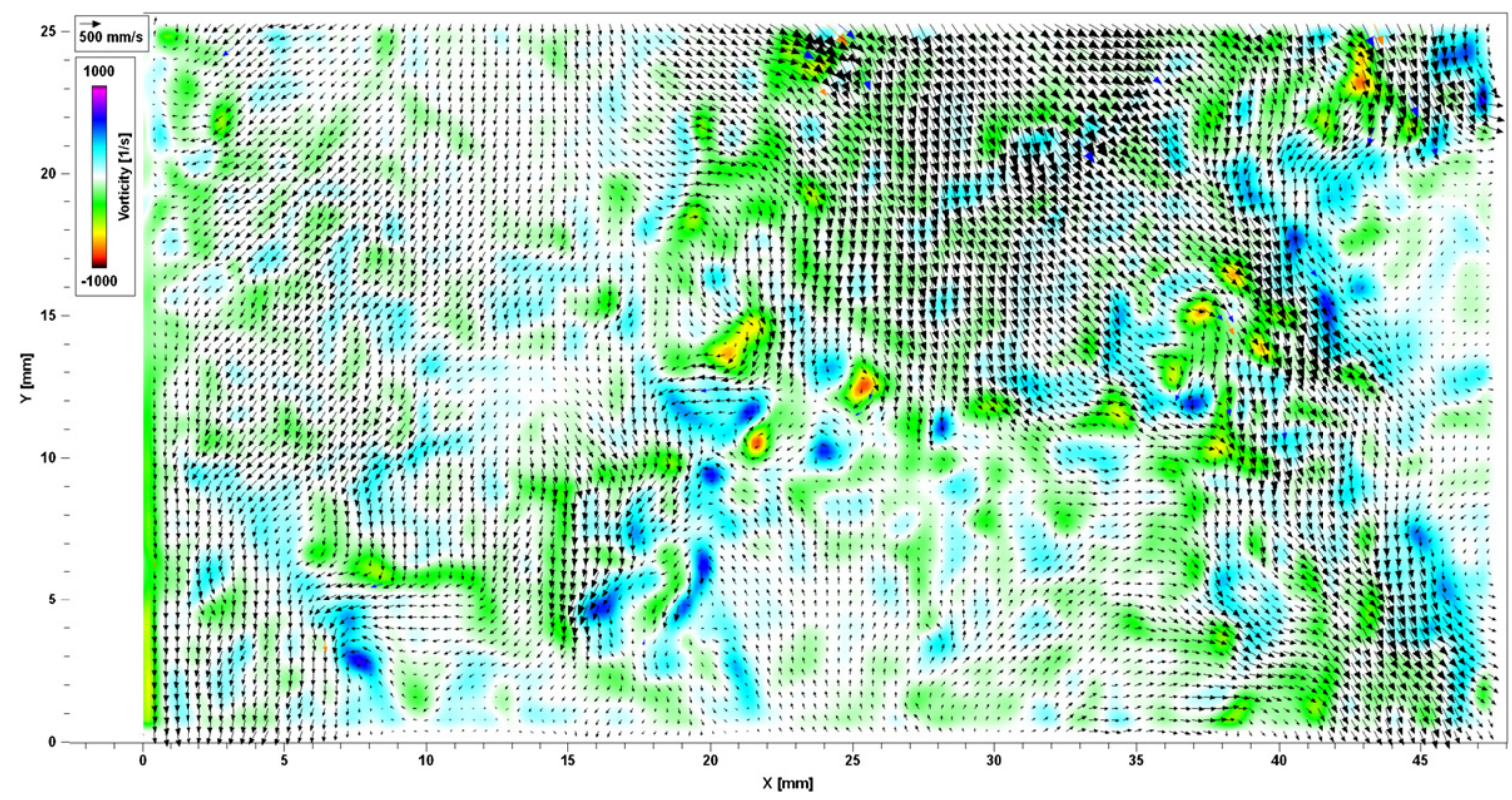

Figure 11. Single PIV map from a time-resolved image sequence acquired at $2 \mathrm{kHz}$ of the turbulent flow downstream of a propeller inside a small water tank. Blue vectors are obtained from lower order correlation peaks while orange vectors indicate interpolated vectors.
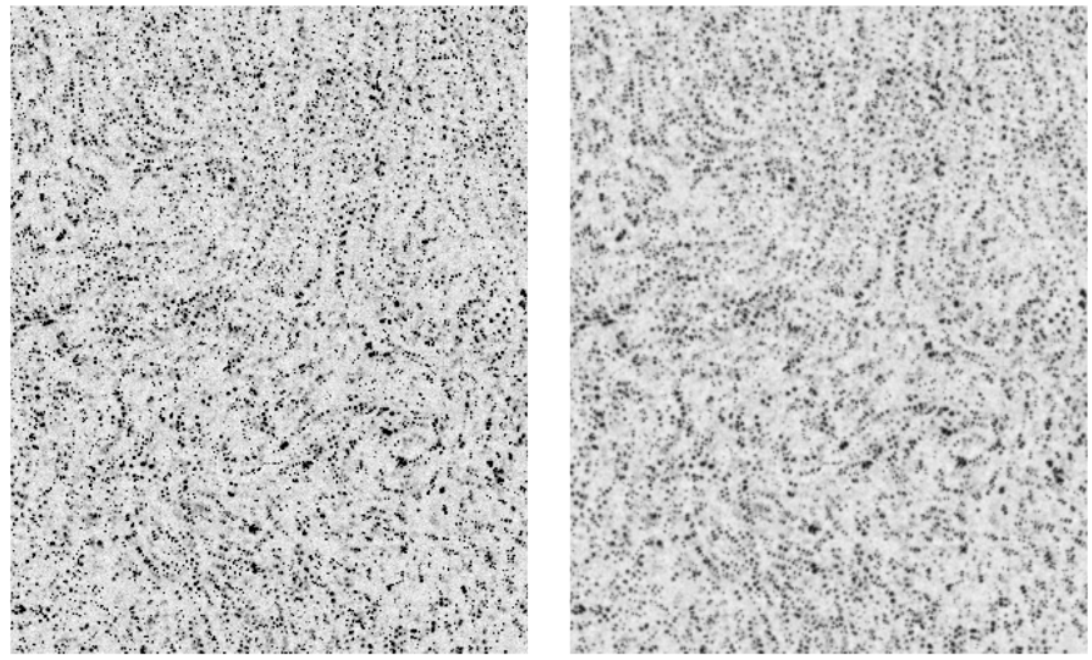

Figure 12. Detail of overlaid PIV recording image pair of water flow in figure 11 with inverted intensity scale (black $=$ bright) extracted at $20 \leqslant x \leqslant 28 \mathrm{~mm}, 7 \leqslant y \leqslant 17 \mathrm{~mm}$. Low-pass-filtered version of the image used for PIV analysis (right). Illumination pulse separation is $\Delta t=500 \mu \mathrm{s}$.
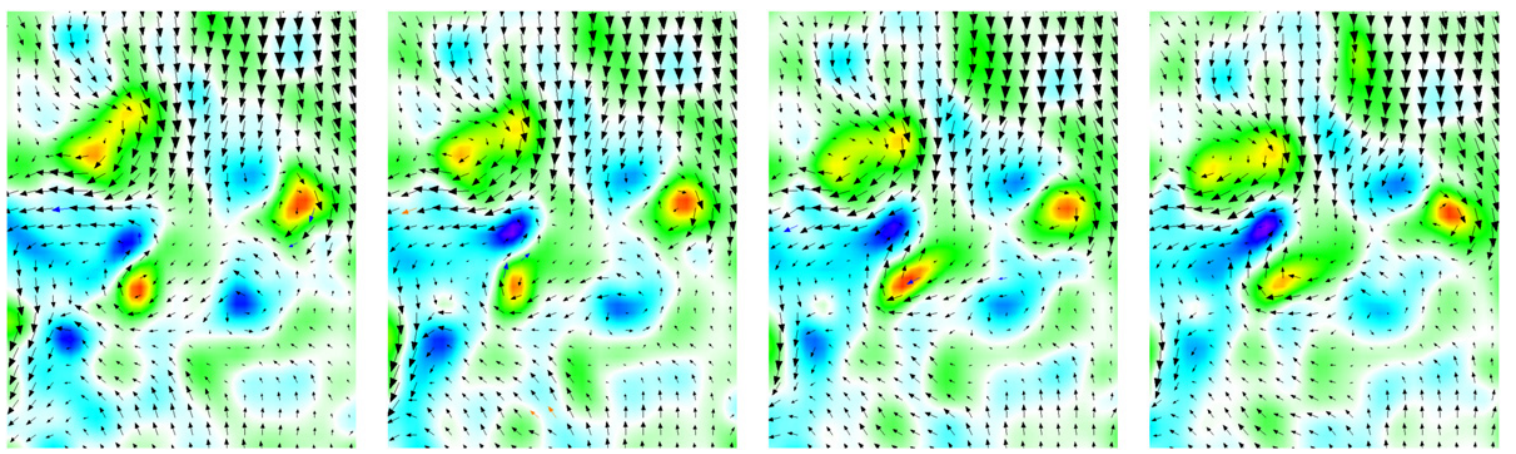

Figure 13. Four consecutive PIV maps of a selected portion of figure 11. Separation between displayed recordings is $1 \mathrm{~ms}$. 

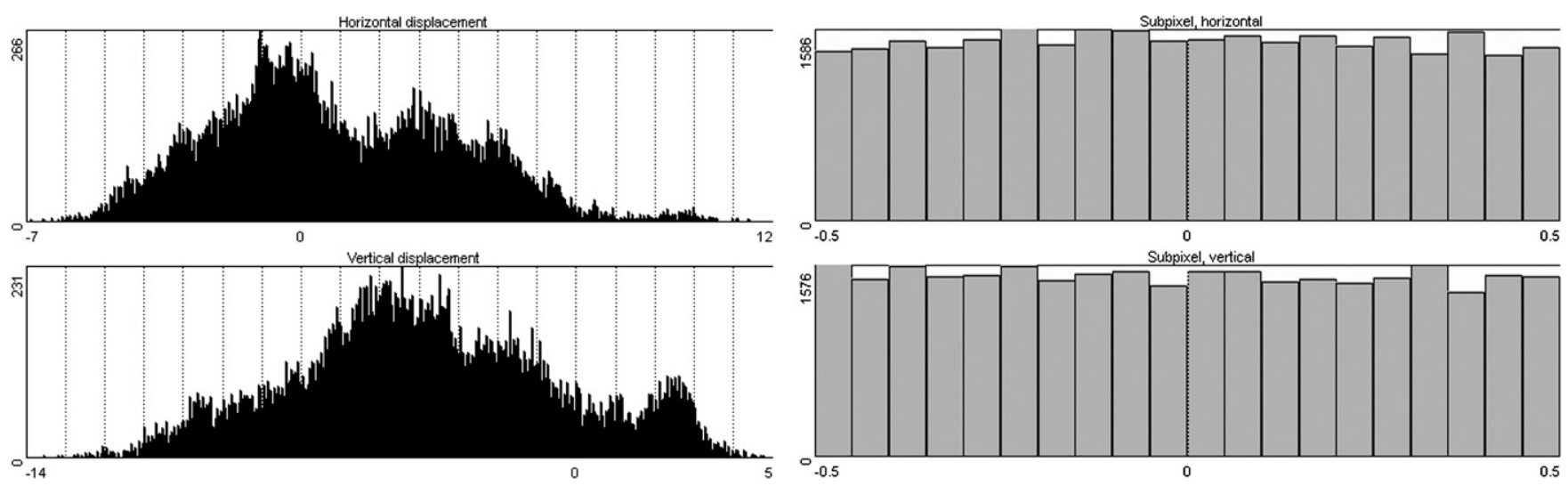

Figure 14. Histograms of the two displacement components of the data provided in figure 11 (left); histograms of fractional displacements (right).

sheet thickness was approximately $1 \mathrm{~mm}$ at the waist widening to about $2 \mathrm{~mm}$ at the edges of the $50 \mathrm{~mm}$ wide imaged area. Current pulses of $50 \mathrm{~A}$ and $\tau_{\mathrm{p}}=20 \mu$ s duration were used, which is more than a factor of 2 below the damage threshold plotted in figure 6. At these settings the LED driver circuits draw an average current of $2 \mathrm{~A}$ at a supply voltage $V_{s} \approx 12 \mathrm{~V}$. Using information on the LED's optical power quoted in the data sheet along with the luminous flux increase provided in figure 4 , the single pulse energy emitted by the LED is estimated to be about $400 \mu \mathrm{J}$.

Prior to PIV processing the recorded images were subjected to image contrast enhancement involving background subtraction and intensity clipping [15]. Additional smoothing of the image with a small kernel further reduced spurious detector noise [16]. Samples of the image data are given in figure 12 before and after image enhancement. The particle image density was sufficient to sample the images with $16 \times 16$ pixel sub-windows, corresponding to $0.4 \times 0.4 \mathrm{~mm}^{2}$. Since the light sheet thickness is of the order of $1 \mathrm{~mm}$ a sample window of $32 \times 32$ pixel was chosen which corresponds to a roughly cubic sampling volume. With these settings the data yield was close to $100 \%$ with about 10-20 spurious vectors per PIV recording consisting of about 7400 vectors. A sequence of an enlarged region of the time-resolved PIV data near $x=20 \mathrm{~mm}$, $y=10 \mathrm{~mm}$ is shown in figure 13 . Each of the image pairs has been processed individually, that is there is no frame-to-frame averaging of the recovered velocity data. Histograms of the recovered displacement data show no signs of peak locking (figure 14).

\section{Discussion}

The previously described experiments clearly demonstrate the viability of using pulsed, high-power LEDs as illumination sources for PIV in classical side-scatter imaging configurations. Generally being an uncollimated light source is one of the more critical drawbacks of the LED. This makes it difficult to establish light sheet illumination commonly found in macroscopic PIV applications unless additional optics such as the fiber optic line lights are used. Unfortunately the large aperture of the typically Lambertian radiative pattern of LEDs (N.A. 1) does not match well to the smaller aperture of optical fibers (typ. N.A. $\approx 0.55$ ), such that a significant amount of light is lost. Comparable measurements between volume illumination and light sheet illumination indicated that up to three quarters of the light is lost by the fiber transmission system. Suitable collimation devices are the subject of ongoing investigations. Nonetheless fiber optic line lights with multiple proximal fiber bundle branches are commercially available and allow the light output to be increased proportionally. The collimating optics for the light sheet can be improved by replacing the plane convex lens of the current setup with a best form lens which reduces the halo around the light sheet.

Collimation is of lesser importance when LEDs are used to provide volume illumination, for instance in microscopic PIV or photogrammetric particle imaging methods such as tomographic PIV or 3D particle tracking. The herein reported PIV measurements of an air flow involved volume illumination in a forward scattering arrangement with the depth of volume defined by the large aperture lens. Due to the small particle size the signal recorded by the camera is rather low. Due to driver limitations these measurements were performed at a rather low drive current of $I_{f}=30 \mathrm{~A}$ which, based on the data in figure 6 , could have increased to $I_{f} \approx 200 \mathrm{~A}$ providing about four times more light. At present, LEDPIV measurements of water flows seem more viable since larger particles in the $10 \mu \mathrm{m}$ range can be used. Successful tomographic PIV measurements of grid turbulence in a water channel have been performed by the authors but are beyond the scope of this paper and will be published in the future.

Due to the quasi-continuous illumination of the flow during the LED pulse, the pulse separation between the two exposures comprising a PIV recording has to be properly matched to avoid streaking. The air flow measurements presented in section 4.1 had a duty cycle of about $13 \%$ ( $2 \mu$ s pulses with $15 \mu$ s separation), which means that for displacements of 15 pixels the streaking is 2 pixels. This is of the same order as the optimum particle image diameter. The self-similarity of the resulting particle image will result in a slightly elongated correlation peak in the direction of 
the displacement vector (ignoring curvature and acceleration effects).

The time-resolved water flow measurements in section 4.2 were acquired at a much smaller duty cycle of 4\% (20 $\mu$ s pulses with $500 \mu$ s period) with which flow speeds in excess of $0.5 \mathrm{~m} \mathrm{~s}^{-1}$ (10 pixel displacement) could be measured reliably in a $50 \times 50 \mathrm{~mm}^{2}$ field of view. A further increase of the duty cycle to $10 \%$ would allow measurements up to $\approx 1.5 \mathrm{~m} \mathrm{~s}^{-1}$ at this magnification without reducing the illumination pulse width.

\section{Conclusions}

The use of LED-based illumination for flow measurement was investigated as a possible alternative to commonly used laserbased illumination. The study was motivated by the recent availability of high-power LEDs with continuous radiant flux levels comparable to that of lasers. Pulsed light at significant intensity levels can be obtained from these solid state devices by briefly overdriving them with high currents. The emitted light was sufficient for PIV measurements in both water and air.

Challenges yet to be solved are associated with the rather wide-angle radiation pattern (i.e., high N.A.) of the light issuing from the luminous surface of the LED. The high numerical aperture restricts the creation of thin light sheets comparable to those achieved with the collimated light of lasers. Nonetheless, reasonable light sheets can be achieved using a fiber optic collimating device with which time-resolved PIV measurements were performed in water at frame rates exceeding $4 \mathrm{kHz}$.

PIV systems using LEDs as light sources can be assembled at significantly reduced costs. The combined cost of LED light source, driver unit components and fiber optics used in the high-speed imaging setup amount to roughly 500.00 Euro. Compact, battery operated systems are feasible and could be of interest for in-flight measurements or autonomous operation in hazardous environments. From a handling point of view the uncollimated light of LEDs is less dangerous, but not necessarily eye-safe, while supply voltages are considerably lower than for pulsed lasers.

In comparison to pulsed lasers commonly used for PIV, which require a pre-trigger time of the order of 100 to $300 \mu \mathrm{s}$ to pump the lasing medium, the light pulses of LEDs can be 'fired' with insignificant delay, that is, within several nanoseconds, depending on the support electronics. The rise time of LEDs is in the $100 \mathrm{~ns}$ range. The pulse repetition rate can be freely varied since LEDs do not need to be operated at specific pulsing frequencies. Pulseto-pulse intensity and spatial variations are practically not present. The broad spectral intensity distribution of the LED's electroluminescence prevents the creation of speckle (interference of coherent light).

Future developments will undoubtedly lead to LEDs with even higher light output per surface area. The high-power devices used for the present investigations had a luminous output of the order $175 \mathrm{~lm}$ per square millimeter of die area $\left(0.3 \mathrm{~W} \mathrm{~mm}^{-2}\right)$ at a luminous efficacy around $20-30 \mathrm{~lm}$ per watt of input power. Luminous efficiencies in excess of $100 \mathrm{~lm} \mathrm{~W} \mathrm{~W}^{-1}$ have already been demonstrated for highpower, white light LEDs. Current technology trends will likely result in LED devices that are suitable light sources for flow diagnostics, in particular for planar techniques as well as the recently introduced volume-resolving, particlebased velocimetry techniques (e.g., tomographic PIV [17] or 3D-PTV [18]). Currently available high-power LEDs are also particularly well suited for applications requiring volume illumination such as shadowgraphy and high-speed schlieren imaging. Beyond the flow imaging applications presented here the use of pulsed LED illumination is also very attractive in high-speed imaging of fast moving objects.

\section{Acknowledgments}

This work was partly funded by the German Ministry of Education and Research (BMBF), PTJ Research Project number 03FPE00003. This support is gratefully acknowledged by the authors.

\section{References}

[1] Hiller W, Lent H M, Meier G E A and Stasicki B 1987 A pulsed light generator for high-speed photography Exp. Fluids 5 141-4

[2] Stasicki B, Hiller W and Meier G E A 1984 Hochfrequenz-Stroboskop mit LED-Lichtquelle Tech. Mess. 51 217-20

[3] Buttsworth D and Ahfock T L 2003 A pulsed LED system for Schlieren flow visualization Tech. Rep. TR-2003-01 (University of Southern Queensland, Australia)

[4] Bröder D and Sommerfeld M 2007 Planar shadow image velocimetry for the analysis of the hydrodynamics in bubbly flows Meas. Sci. Technol. 18 2513-28

[5] Lindken R and Merzkirch W 2002 A novel PIV technique for measurements in multiphase flows and its application to two-phase bubbly flows Exp. Fluids 33 814-25

[6] Nogueira S, Sousa R, Pinto A, Riethmuller M and Campos J 2003 Simultaneous PIV and pulsed shadow technique in slug flow: a solution for optical problems Exp. Fluids 35 598-609

[7] Estevadeordal J and Goss L 2005 PIV with LED: Particle Shadow Velocimetry (PSV) technique 43rd AIAA Aerospace Sciences Meeting and Exhibit (Reno, NV)

[8] Chételat O and Kim K C 2002 Miniature particle image velocimetry system with LED in-line illumination Meas. Sci. Technol. 13 1006-13

[9] Hagsäter S M, Westergaard C H, Bruus H and Kutter J P 2003 Investigations on LED illumination for micro-PIV including a novel front-lit configuration Exp. Fluids 44 211-9

[10] Willert C, Freitag S and Hassa C 2008 High speed imaging of fuel sprays using a low-cost illumination source 22nd European Conf. on Liquid Atomization and Spray Systems (ILASS 2008)

[11] Charvat A, Stasicki B and Abel B 2006 Product screening of fast reactions in IR-laser-heated liquid water filaments in vacuum by mass spectrometry J. Phys. Chem. A $1103297-306$

[12] Stasicki B, Abel B, Charvat A and Faubel M 2005 Visualization of laser-induced liquid micro-jet disintegration by means of high-speed video stroboscope 26th Int. Congr. on High Speed Photography and Photonics 
(Alexandria, VA, USA), Proc. SPIE vol 5580, ed D L Paisley, S Kleinfelder, D R Snyder and B J Thompson pp 335-46

[13] Luminus Devices Inc. 2008 Technical Product Data Sheet PDS-001226: CBT-120 Series, PhlatLight LED Illumination Products

[14] Saleh B and Teich M 2007 Fundamental of Photonics 2nd edn (Hoboken, NJ: Wiley)

[15] Shavit U, Lowe R J and Steinbuck J V 2007 Intensity capping: a simple method to improve cross-correlation PIV results Exp. Fluids $\mathbf{4 2} 225-40$
[16] Raffel M, Willert C E, Wereley S T and Kompenhans J 2007 Particle Image Velocimetry-A Practical Guide 2nd edn (Berlin: Springer)

[17] Elsinga G, Scarano F, Wieneke B and van Oudheusden B 2006 Tomographic particle image velocimetry Exp. Fluids 41 933-47

[18] Pereira F, Gharib M, Dabiri D and Modarress D 2000 Defocusing digital particle image velocimetry: a 3-component 3-dimensional DPIV measurement technique: application to bubbly flows Exp. Fluids 29 S078-84 